\title{
CAMAR 2.0: Future Direction of Context-Aware Mobile Augmented Reality
}

\author{
Choonsung Shin, Wonwoo Lee, Youngjung Suh, Hyoseok Yoon, Youngho Lee and \\ Woontack Woo \\ GIST U-VR Lab. \\ \{cshin,wlee, ysuh, hyoon, ylee,wwoo\}@gist.ac.kr
}

\begin{abstract}
With the rapid spreading of ubiComp and mobile augmented reality, the interaction of mobile users in $U-V R$ environments has been evolving. However, current interaction is limited to individuals' experiences with given contents and services. In this paper, we propose CAMAR 2.0 as a future direction of CAMAR aiming at improving perception and interaction of users in U-VR environments. We thus introduce three principles for future interaction and experience in $U-V R$ environments. We also discuss technical challenges and promising scenarios for realizing the vision of CAMAR 2.0 in $U-V R$ environments. ${ }^{*}$
\end{abstract}

\section{Introduction}

With emergence of Ubiquitous Virtual Reality (UVR), the interactions of users with contents and services have being changing radically [1]. The aim of U-VR is to increase the quality of human life by providing users with personalized services and contents according to social human activity in mixed reality environments [2]. The contents in U-VR environments also have been evolving with intelligence, realism and mobility [3]. Furthermore, as T. O'Reilly emphasized Web 2.0 as the next paradigm of current Web users' contribution and participation have a great attention from various areas [4]. Similarly to Metaverse, users are allowed to freely create and share information and contents with others any where at any time [5]. Hence, users need a novel user interface which allows them to holistically exploit the benefits of contents and services in U-VR environments.

A lot of researches have been trying to realize a new interaction method by combining context-awareness and mobile augmented reality. The first generation of mobile AR using context-awareness was based on

\footnotetext{
* This research is supported by KOCCA of MCST, Korea, under the CT R\&D Program 2009.
}

laptop and mainly used location information as a context [6-7]. Afterward, the convergence of contextawareness and mobile augmented reality have been shifted to light weight platforms such as PDAs, ultra mobile personal computers (UMPCs) and mobile phones [8-10]. Furthermore, others tried to use a domain knowledge and behavior model to improve interaction in mobile augmented reality [11-12].

Although the previous approaches showed the importance of the convergence of context-awareness and mobile augment reality, they still have limitation in allowing users to take advantages over U-VR environments and contents. First, most of work focused on direct use of low-level context obtained from sensors such as GPS and accelerometers. Second, the interaction limited to particular objects or markers makes it challenging to enable users to interact with rich information and contents over different objects. Third, users' participation in creating contents and services were limited since they used given contents stored in a server or their mobile devices, and services developed by experts.

In order to tackle those limitations and exploit benefits of the previous work, we propose CAMAR 2.0 as a future direction of Context-aware mobile AR In CAMAR 2.0, we consider users as coauthors of contents and co-developers of services and applications in U-VR environments. We thus initiate a set of principles supporting CAMAR 2.0 to meet the needs of users in U-VR environments. In addition, various technical challenges and useful scenarios are elaborate the principles of CAMAR 2.0.

This paper is organized as follows. We first propose CAMAR 2.0 with three principles. We then describe various technical challenges in realizing the vision of CAMAR 2.0. Promising scenarios are introduced to argue possibility and usefulness of CAMAR 2.0. Finally, we conclude with future work.

\section{CAMAR 2.0}

We proposed Context-Aware Mobile Augmented Reality (CAMAR) for personalization, selective sharing and interaction of contents in ubiquitous smart 
spaces [13][14]. It mainly focused on how to combine context-awareness and augmented reality in a mobile platform. However, the previous implementations have several limitations in satisfying demanding users in UVR environments. Similar to the previous work, main limitation was the lack of user's participation. Users were only enabled to interact with contents and services specified by developers or service providers.

In order to overcome the limitations, we introduce a concept of CAMAR 2.0 as a future direction of CAMAR. Enhancing the previous approaches, the next CAMAR turns into users' ability and sustainable participation with others in U-VR environments. We define CAMAR 2.0 as a convergence technology that is aiming at realizing sustainable, evolutional smart space through active participation, social sharing and seamless collaboration of users by contextually authoring and augmenting relevant contents over real objects in mobile environments. In order to achieve this vision of CAMAR 2.0, there are three principles: ubiquitous augmentation, high-level context-awareness and sustainable participation. The ubiquitous augmentation allows us to interact with objects with pervasive rich information in various smart spaces. The high-level context-awareness enables us to transparently and seamlessly interact with the desired objects, information and users. The sustainable participation motivates us to be a member of the society as a co-developer of applications and services and a coauthor of contents and information associated with objects. Here are the details of the principles in CAMAR 2.0.

Ubiquitous augmentation. People usually spend a lot of time with objects existing in our daily life. The real space and objects are associated with a corresponding virtual space and objects. In such environment, users' perception and interaction can be improved when they are allowed to interact with the objects combined with relevant information. By recognizing and tracking entities and space ubiquitously in U-VR environments, users can interact with the objects with rich information. By merging real space and objects with virtual space and objects, users can exploit benefits of both spaces in any where at any time.

High-level context-awareness. There are a huge amount of information existing in the real space and the virtual space. However, users want to interact with selective entities among the available information, contents, services and applications. In order to augment relevant information, it is important to understand users and their situations for preventing a seam and providing transparency from augmentation. Therefore, more high-level understanding of users is required in terms of environmental and user aspect. An environment can be interpreted by merging various sensing information from different sources such as illuminators, accelerometers, compass, to improve the quality of augmentation and tracking of objects. In the aspect of users, social and cultural context such as social relationship, cultural background and etc., enhances the quality of augmentation and interaction.

Sustainable participation. The ability of individuals is limited to a relatively small area and a given infrastructure slowly evolves on demand. In order to broaden their experience and knowledge in such environments, a multitude of people needs to continuously participate in their society. By sustainably creating and extending the services and contents, they are able to express their opinion and creativity to already existing information and knowledge. They are also able to take advantages over rich information from diverse sources and cultures by collaborating with others through various activity and work and by sharing context, contents and services in U-VR environments.

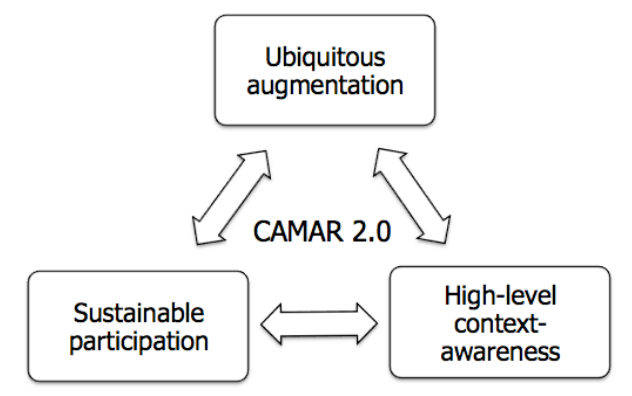

Figure 1. Relationship of the principles

Those principles also compensate each other to improve experience and interaction of users in U-VR environments. Context-awareness accelerates the participation and ubiquitous augmentation by connecting relevant users and information on the behalf of users. The participation enriches contextawareness and augmentation by creating information and services and by working together. The augmentation contributes to improving the awareness of users and participation with augmenting information over the real objects and space. Therefore, U-VR environments are able to evolve with the continuous interaction of principles of CAMAR 2.0.

\section{Technical Challenges}

There are a number of technical challenges to realize CAMAR 2.0. Some of them are covered by the technologies in other research areas but needs to be combined and tailored to lightweight mobile platforms, and others should be developed concerning context awareness and mobile augment reality. 
Hybrid multi-object tracking and recognition. There are various types of objects in indoor and outdoor environments. However, any single method of tracking and recognition technology could not be applied for different objects. Thus, the various technologies should be combined with other strategies to stably augment contents on various existing objects. These combined methods should also dynamically and efficiently support the tracking and recognition according to types of objects and situation.

Coordination and Registration of Real \& Virtual Space. As a real space is associated with its VR space, real objects are also related to virtual objects and information in the VR space. Therefore, a coordination method is required to combine two spaces and registration is needed to augment the information and virtual objects over the real space and objects.

Sensing and reasoning. More high-level contexts about how a user interacts with an environment and other users should be derived. For this purpose, sensing technologies are required to monitor changes of users and their surroundings by adopting various mechanisms. Reasoning technologies are needed to infer the meaning of context by merging and integrating sensory information obtained from various sensors and understanding interaction history, social and cultural diversity.

Intelligent visualization. While huge amount of information is available, mobile platforms have a small screen size and only limited information is already related to users. In order to recommend the right information to the right person, information-filtering technologies are also combined with high-level context associated with mobile users. In addition, the contents augmented on real objects need to intelligently behave by understanding a user and his/her situation. For this purpose, a behavior model that understands context is required for generating responses of the contents.

AR in-Situ Mash-up. While users move, they want to create services and contents associated with their interested area and objects. Therefore, relevant interaction interface and services associated with the objects are discovered from various sources. The protocols and interaction methods to combine services from different sources should be defined and developed. In order to allow users to freely create and distribute the contents, authoring, tagging and retrieval technologies are developed by exploiting mobile augmented reality situations.

Mobile social network and mediation. Users need to share their information and contents with selected entities. However, they should concern privacy and intimacy to effectively share and collaborate with others. Mobile social network can be use to understand social structure by exploiting profile and proximity sensing of nearby devices and users in the real and virtual spaces. Mobile social mediation is also required for effectively utilizing the social network by reducing a social gap between users.

Open dynamic framework. While many technologies coexist and evolve, applications should be developed without concerning details and changes of the technologies. Therefore, an open software framework that abstracts functionality from complex technologies by providing open APIs, is required for developing new applications. It should also dynamically compose required technologies in Plug and Play fashion when a particular functionality is required to better suite limited resources of mobile platforms.

\section{Possible Applications}

While numerous of applications can be developed based on CAMAR 2.0, we introduce a smart space assistant and tour guide widely used by general users.

\subsection{Smart Space Assistant}

One of the interesting applications in mobile augmented reality is a smart space assistant where many services and devices readily available to use. Most pervious work focused on augmentation over the smart objects. On the other hand, our smart space assistant based on CAMAR 2.0 improves the interaction and experience in the smart space in several ways.

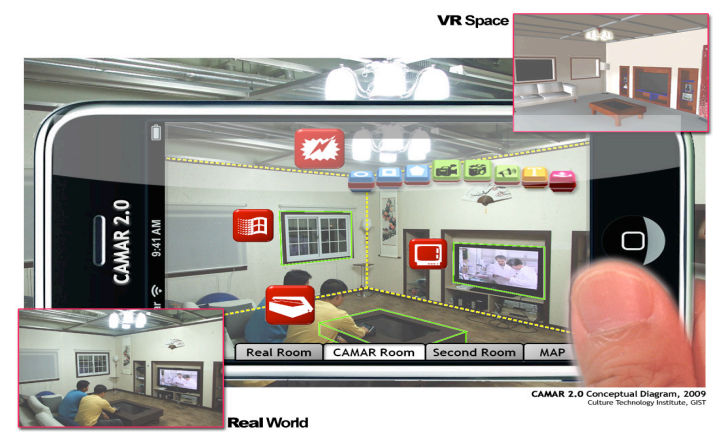

Figure 2. Smart home assistant

As shown in Figure 2, users can view the smart space and find sensors and applications by merging a real smart space with its corresponding virtual smart space. Therefore, the users can control and manage the applications and sensors in an intuitive way. In addition, users also can modify and add desired functions of smart objects and applications. They also can post a message on an object or a certain area within the smart space and share the message with other users in different location via virtual space. With user's 
participation and collaboration with others, the smart space assistant contributes to allow the smart space to continuously evolve to meet the user's demands and preferences.

\subsection{Tour Guide 2.0}

One of the interesting applications in mobile augmented reality is a tour guide because users need to know their surroundings to find a right way and information. Our tour guide applications based on CAMAR 2.0 are different from the previous applications because users can contribute to enrich the guidance system by the principles and technologies.

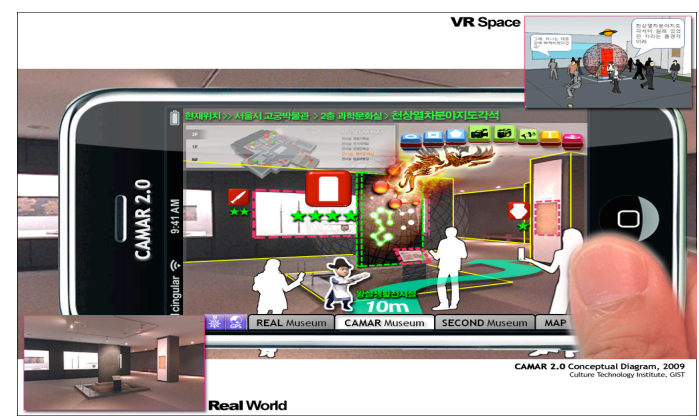

(a)

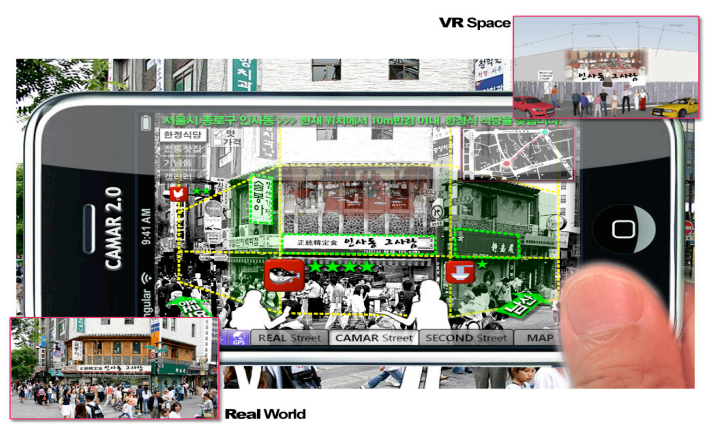

(b)

Figure 3. Tour guides based on CAMAR 2.0. An indoor tour guide (a) and outdoor tour guide (b).

As shown in both Figure 3(a) and (b), users are not only are guided with augmented information and contents in the real space through a mobile device. In the scenarios, various objects in the real space are considered as tags for augmentation. The contents are the given services and knowledgeable information offered by various sources including a corresponding VR space. The users are enabled to experience rich augmented information from various sources to understand their surrounding. The users are also allowed to extend and mashup the existing contents for inspiring his/her experience and knowledge over the exiting contents. Furthermore, the newly created contents are shared by other users based on their social network and context. With continuous consumption and reproduction of contents and services, it is expected that users exploit and contribute to tour areas with abundance of collective and accumulated information and collaboration with other users through CAMAR 2.0.

\section{Conclusion}

As a computing paradigm has been changing with emerging U-VR and contents, user interaction and experience also require evolution. For this purpose, we proposed CAMAR 2.0 empowered by circulation of ubiquitous augmentation, high-level context-awareness and sustainable participation. Therefore, CAMAR 2.0 enhances the interaction and experience of users not only by enabling them to express opinion and extend their knowledge and but also by bridging individuals into a member of the society.

\section{References}

[1] S. Kim, Y. Suh, Y. Lee and W.Woo, "Toward ubiquitous VR: When VR Meets ubiComp," In Proc of ISUVR 2006, pp. 1-4.

[2] Y. Lee, S. Oh, C. Shin and W. Woo, "Ubiquitous Virtual Reality and Its Key Dimension,” In Proc. of IWUVR 2009, pp. 5-8.

[3] K.Kim, S. Oh, J.Han and W. Woo, "u-Contents: Describing Contents in an Emerging Ubiquitous Virtual Reality," In Proc. of IWUVR 2009, pp. 9-12.

[4] T. O'Reilly, "What Is Web 2.0 - Design Patterns and Business Models for the next Generation of Software," http://ww.oreillynet.com/pub/a/oreilly/time/news/2005/09/30/what-isweb-20.html.

[5] J. Smart, J. Cascio and J. Paffendorf, "Metaverse Roadmap Overview," 2007.

[6] T. Höllerer, S. Feiner, T. Terauchi, G. Rashid and D. Hallaway, "Exploring MARS: Developing Indoor and Outdoor User Interfaces to a Mobile Augmented Reality System," Computers and Graphics, 23(6), Elsevier Publishers, Dec. 1999, pp. 779-785.

http://research.nokia.com/research/projects/mara/index.html [7] M. Bauer, B. Bruegge, G. Klinker, A. MacWilliams, T. Reicher, S. Riß, C. Sandor and M. Wagner, "Design of a Component-Based Augmented Reality Framework," In Proc. of ISAR 2001.

[8] Henrysson and Ollila, "UMAR - Ubiquitous Mobile Augmented Reality," In Proc. of MUE, pp. 41-45, 2004

[9] Mobile Augmented Reality Applications project, http://research.nokia.com/research/projects/mara/index.html

[10] D. Schmalstieg and D. Wagner, "Experiences with Handheld Augmented Reality," In Proc. of ISMAR 2007, pp. 3-15.

[11] I. Barakonyi and D. Schmalstieg, "Ubiquitous Animated Agents for Augmented Reality," In Proc. of ISMAR 2006, pp. 145-154.

[12] J. Lee, D. Seo, and G. Rhee, "Visualization and interaction of pervasive services using context-aware augmented reality," Expert Systems with Applications, vol. 35, no. 4, pp. 1873- 1882, 2008.

[13] Y. Suh, Y. Park, H. Yoon, Y. Chang, and W. Woo, "Context-Aware Mobile AR System for Personalization, Selective Sharing, and Interaction of Contents in Ubiquitous Computing Environments," in Proc. Of HCII, LNCS 4551, pp. 966-974, 2007.

[14] S. Oh and W. Woo, "CAMAR: Context-aware Mobile Augmented Reality in Smart Space," In Proc. of IWUVR 2009, pp. 48-51. 